\title{
Effect of extracorporeal shock-wave therapy on spasticity after central nervous system injury: A Meta-analysis
}

\section{Po-Cheng Hsu, Pin-Yi Wu, Tsui-Fen Yang, Chen-Liang Chou \\ Department of Physical Medicine and Rehabilitation, Taipei Veterans General Hospital -Taipei, Taiwan}

Introduction: Spasticity is a common complication of upper motor neuron lesions and major cause of disability, affecting health-related quality of life. Extracorporeal shock-wave therapy (ESWT) has become a new treatment modality for spasticity and is considered a safe intervention.

Purpose: To investigate the effectiveness of ESWT in treating patients with spasticity after upper motor neuron lesions, a meta-analysis of controlled trials were performed to evaluate changes in Modified Ashworth Scale scores.

Methods: Comprehensive electronic searches were conducted using PubMed, Embase, Cochrane Controlled Trials Register, Cochrane, and other databases. Controlled trials conducted before October 2017, which were restricted only to those reported in English, were included. The study selection, quality of studies, and data extraction were conducted by two reviewers independently. Disagreements were settled by consulting a third reviewer to reach a consensus.

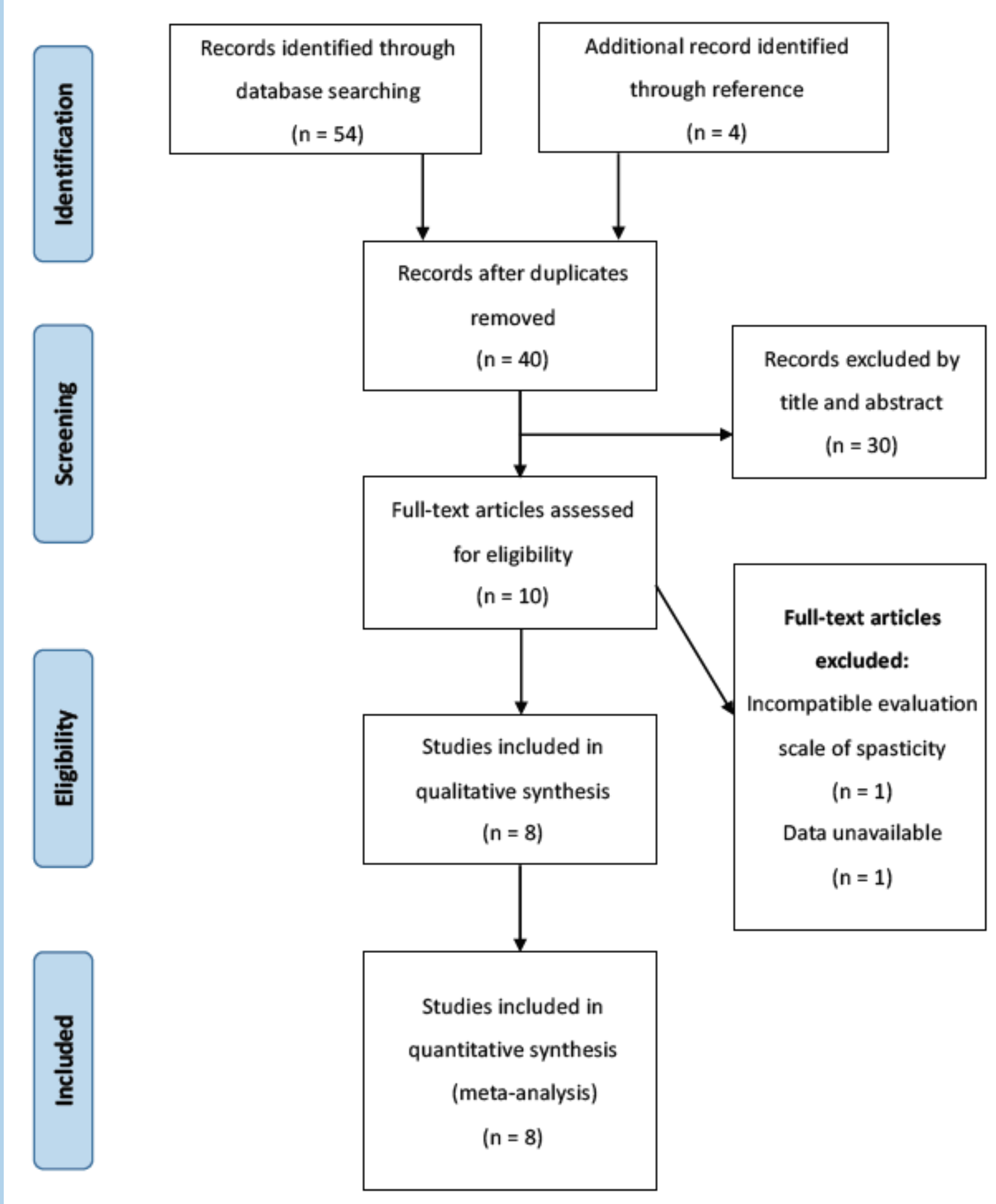

\section{Results:}

Eight trials with 270 subjects (four in stroke, three in cerebral palsy, and one in multiple sclerosis) fulfilled the inclusion criteria. Meta-analysis showed that ESWT significantly reduced spasticity immediately (SMD -4.0: 95\% confidence interval [CI], -2.37 to -2.76 ; I2 $=95 \%$ ), 4 weeks (SMD -2.51 : $95 \% \mathrm{CI}$, -3.40 to $-1.62 ; \mathrm{I} 2=93 \%$ ), and 12 weeks (SMD $-1.92(95 \% \mathrm{CI},-1.92$ to $-0.95 ; \mathrm{I} 2=72 \%)$ after intervention
Further subgroup analysis demonstrated the effects in different disease types, parts treated, and radial/focus ESWT. Treatment with $\geq 3$ sessions of ESWT showed better response in spasticity at 12 weeks. The optimal protocol could not be identified.
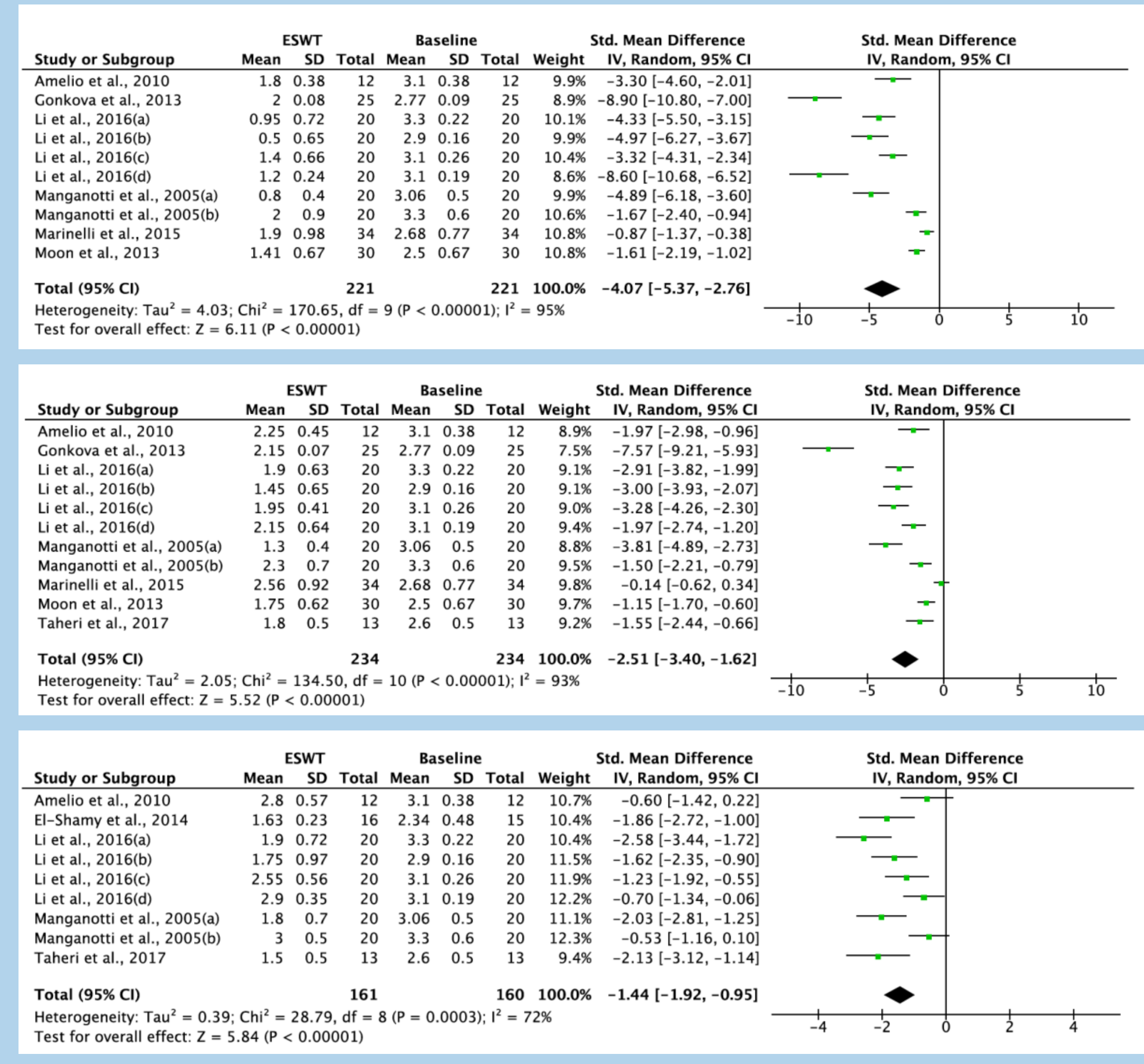

Subgroup analysis of the pooled SMD in reducing spasticity immediately after and 4 and 12 weeks after ESWT

\begin{tabular}{|llll|}
\hline $\begin{array}{l}\text { Subgroup } \\
\text { Session }\end{array}$ & $\begin{array}{l}\text { Pooled SMD } \\
\text { immediately }\end{array}$ & $\begin{array}{l}\text { Pooled SMD at } \\
\text { Week 4 }\end{array}$ & $\begin{array}{l}\text { Pooled SMD at } \\
\text { Week I2 }\end{array}$ \\
\hline I session & $-4.42(-6.09--2.76)$ & $-2.89(-4.03--1.75)$ & $-0.97(-1.28--0.66)$ \\
\hline$>3$ session & $-3.35(-6.25--0.44)$ & $-1.37(-2.24--0.51)$ & $-1.63(-2.10--1.15)$ \\
\hline
\end{tabular}

Type of ESWT

\begin{tabular}{llll}
\hline Focus & $-2.76(-4.06--1.45)$ & $-1.92(-2.72--1.12)$ & $-1.39(-2.11--0.67)$ \\
\hline Radial & $-5.05(-7.46--2.65)$ & $-2.34(-3.45--1.23)$ & $-1.32(-1.75--0.88)$ \\
\hline Part & & & \\
\hline Upper & $-4.48(-6.09--2.88)$ & $-2.69(-3.40--1.98)$ & $-1.4 I(-2.02--0.8 I)$ \\
\hline Lower & $-3.43(-5.45--1.4 I)$ & $-1.66(-2.58--0.73)$ & $-1.26(-1.70--0.8 I)$
\end{tabular}

NOTE. Values are expressed by their point estimate with a $95 \% \mathbf{C l}$.

\section{Conclusions:}

The present meta-analysis revealed that ESWT effectively alleviates spasticity in patients after upper motor neuron lesions, regardless of disease type and parts treated. Both radial and focus ESWTs could decrease spasticity, and treatment with $\geq 3$ sessions of ESWT was more effective than that with one session at 12 weeks. Moreover, no serious side effects were observed after ESWT. 